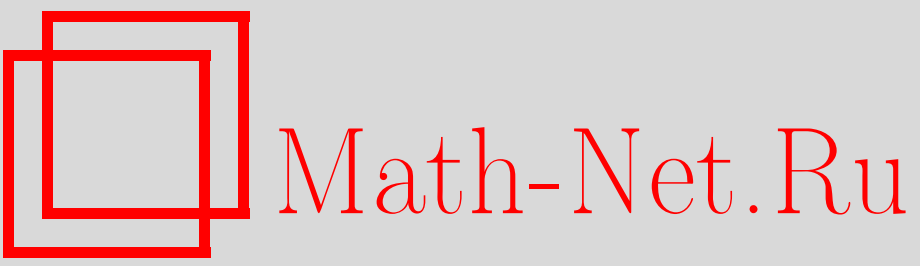

М. М. Анзин, О плотности решетчатого покрытия для $n=11$ и $n=14, \mathrm{YMH}$, 2002, том 57, выпуск 2, 187-188

DOI: https://doi.org/10.4213/rm501

Использование Общероссийского математического портала Math-Net.Ru подразумевает, что вы прочитали и согласны с пользовательским соглашением

http://www.mathnet.ru/rus/agreement

Параметры загрузки:

IP : 54.198 .55 .26

26 апреля 2023 г., 05:54:28 


\title{
О ПЛОТНОСТИ РЕШЕТЧАТОГО ПОКРЫТИЯ ДЛЯ $n=11$ И $n=14$
}

\author{
М. М. Анзин
}

1. Основной результат и история вопроса. Задача о наименее плотных решетчатых покрытиях (обзор см. в [1]) состоит в отыскании для каждой размерности $n$ такой решетки $\Gamma_{n}$, которая дает наименьшее значение плотности $\theta_{n}(\Gamma)$ решетчатого покрытия евклидова пространства $E^{n}$.

В настоящей работе мы сводим исследование функции $\theta_{n}(\Gamma)$ к исследованию функции $\eta(\Gamma)-$ аналога функции Эрмита

$$
\eta(\Gamma)=\eta\left(f_{\Gamma}\right)=D^{2} / \sqrt[n]{\operatorname{det} f_{\Gamma}}=(2 R)^{2} / \sqrt[n]{\operatorname{det} f_{\Gamma}},
$$

где $D=D(\Gamma)=2 R$ - диаметр шара покрытия, $\operatorname{det} f_{\Gamma}-$ определитель матрицы положителшной квадратичной формы $f_{\Gamma}$, отвечающей некоторому основному реперу решетки $\Gamma$. Если $\Omega_{n}-$ объем $n$-мерного шара единичного радиуса, то $\eta(\Gamma)=4\left(\theta_{n}(\Gamma) / \Omega_{n}\right)^{2 / n}$.

$\mathrm{K}$ настоящему времени задача о наименее плотных решетчатых покрытиях полностью решена только для размерностей $n=2,3,4,5$ (подробности см. в [1]). Для других $n$ известны только оценки. Во всех перечисленных случаях решение достигается на решетках $A_{n}^{*}$, которым отвечают квадратичные формы из серии "главной формы первого типа Вороного"

$$
\sum_{i=1}^{n} n x_{i}^{2}-\sum_{\substack{i, j=1 \\ i \neq j}}^{n} x_{i} x_{j}, \quad \text { для которых } \eta\left(A_{n}^{*}\right)=\frac{n(n+2)}{3 n+3} \sqrt[n]{n+1}
$$

Существует гипотеза, что решетка Лича $\Lambda_{24}$ дает абсолютньй минимум функции $\eta(\Gamma)$ для $n=24: \eta\left(\Lambda_{24}\right)=8<\eta\left(A_{24}^{*}\right)=9.514 \ldots$ Оценки функции $\eta(\Gamma)$, лучшие (меньшие), чем $\eta\left(A_{n}^{*}\right)$, были найдены для всех размерностей $n>24$ в работах [2] и [3]; для $5<n<24$ такие оценки известны толшко для размерностей $n=22,23$ [4] и $n=9$ [5].

Основная теорема. Имеют место соотношения

$$
\begin{aligned}
& \eta_{11} \leqslant \eta\left(A_{11}^{4}\right)=4.875 \ldots<\eta\left(A_{11}^{*}\right)=4.978 \ldots, \\
& \eta_{14} \leqslant \eta\left(A_{14}^{5}\right)=5.891 \ldots<\eta\left(A_{14}^{*}\right)=6.040 \ldots,
\end{aligned}
$$

əде $\eta_{n}=\inf _{\Gamma \subset E^{n}} \eta(\Gamma)=\min _{\Gamma \subset E^{n}} \eta(\Gamma), A_{11}^{4} u A_{14}^{5}-$ решетки Kоксетера (cм. [6]).

2. Полное описание $L$-разбиений решеток Коксетера $A_{11}^{4}$ и $A_{14}^{5}$. Формы $A_{11}^{4}$ и $A_{14}^{5}$ имеют характеристики: арифметический минимум $\min A_{11}^{4}=1, \min A_{14}^{5}=1$; число пар минималшных векторов $s\left(A_{11}^{4}\right)=66, s\left(A_{14}^{5}\right)=105 ;$ определитель $\operatorname{det} A_{11}^{4}=3 / 2^{13}, \operatorname{det} A_{14}^{5}=$ $3 /\left(5 \cdot 2^{14}\right)$.

УтвеРЖДЕнИЕ 1. L-разбиение решетки $A_{11}^{4}$ образовано многогранниками, конгруэнтными 16 попарно неэквивалентным L-телам, характеристики которых указаны в таблице. Максимальное значение радиуса L-тел достигается на теле $L_{3}$ и квадрат радиуса решетчатого покрытия равен $R^{2}\left(A_{11}^{4}\right)=19 / 32$. Тем самым $\eta\left(A_{11}^{4}\right)=19 / 4$. $\sqrt[11]{4 / 3}=4.875 \ldots$

УТВеРЖДЕнИЕ 2. L-разбиение решетки $A_{14}^{5}$ образовано многогранниками, конгруэнтными 31 попарно неэквивалентным L-телам. Максимальное значение квадрата радиуса L-тел (т.е. квадрат радиуса решетчатого покрытия) равно $R^{2}\left(A_{14}^{5}\right)=71 / 100$. Тем самым $\eta\left(A_{14}^{5}\right)=142 / 25 \cdot \sqrt[14]{5 / 3}=5.891 \ldots$.

Работа выполнена при поддержке Российского фонда фундаменталшных исследований (грант № 00-01-00619). 
3. Пояснения к таблице. В таблице в граффе "L" приводятся "технические" номера $L$-тел (от 0 до 15). В графе "8 $\mathbf{R}^{2}$ " указаны квадраты радиусов шаров, описанных вокруг $L$-тел, умноженные на 8. В графе "V" указано количество вершин $L$-тела. Обозначения в графе "Описание", означают следующее.

" $p(n+1)$ " - $n$-мерный симплекс, состоящий из $n+1$ вершины.

" $O(k)+p(t)$ ", где $k+t=n,-$ " $t$-этажная" пирамида, построенная над $k$-мерным ортаэдром.

" $O(n)$ " - $n$-мерный ортаэдр.

"O(3) $+p(9) "$ - тело, являющееся выпуклой оболочкой 3-мерного ортаэдра (т.е. октаэдра) и добавленных к нему 9 вершин.

"O(5) $+O(5) "$ - тело, состоящее из двух разнесенных в пространстве ортаэдров размерностей 5 и 5 . Отметим, что именно это $L$-тело имеет максимальный радиус в размерности $n=11$, совпадающий с радиусом решетчатого покрытия.

Для исследованных решеток построены схемы соседства (классов) $L$-тел. В случае $n=11$ соседство многогранников (вида) $L_{4}$ с $L_{7}$ осуществляется по двум попарно неэквивалентным классам гиперграней. В случае $n=14$ для пяти пар (классов) многогранников соседство осуществляется по двум попарно неэквивалентным классам гиперграней, а для одной пары классов многогранников соседство осуществляется по трем попарно неэквивалентным классам гиперграней. В остальных случаях соседства осуществляются по попарно эквивалентным гиперграням. В заключение отметим, что в обоих рассматриваемых случаях встречаются правильные симплексы. Их относительные объемы равны 4 и 5 соответственно. Это заранее известные $L$-тела (см. [7]), с которых мы и начинали перечисление всех $L$-тел.

\begin{tabular}{|c|c|c|c||c|c|c|c|}
\hline $\mathbf{L}$ & $8 \mathbf{R}^{2}$ & $\mathbf{V}$ & Описание & $\mathbf{L}$ & $8 \mathbf{R}^{2}$ & $\mathbf{V}$ & Описание \\
\hline$L 0$ & $3 \frac{2}{3}$ & 12 & $p(12)$ & $L 12$ & $4 \frac{5}{16}$ & 14 & $O(3)+p(8)$ \\
\hline$L 6$ & $4 \frac{5}{12}$ & 12 & $p(12)$ & $L 14$ & $4 \frac{1}{6}$ & 14 & $O(3)+p(8)$ \\
\hline$L 9$ & $4 \frac{2}{3}$ & 12 & $p(12)$ & $L 2$ & $4 \frac{67}{100}$ & 16 & $O(5)+p(6)$ \\
\hline$L 10$ & $4 \frac{305}{588}$ & 12 & $p(12)$ & $L 4$ & $4 \frac{5}{8}$ & 16 & $O(5)+p(6)$ \\
\hline$L 13$ & $4 \frac{1}{4}$ & 12 & $p(12)$ & $L 7$ & $4 \frac{5}{8}$ & 16 & $O(5)+p(6)$ \\
\hline$L 15$ & $3 \frac{263}{300}$ & 12 & $p(12)$ & $L 1$ & $4 \frac{1}{4}$ & 22 & $O(11)$ \\
\hline$L 5$ & $4 \frac{23}{48}$ & 14 & $O(3)+p(8)$ & $L 8$ & $4 \frac{1}{2}$ & 15 & $O(3)+p(9)$ \\
\hline$L 11$ & $4 \frac{5}{12}$ & 14 & $O(3)+p(8)$ & $L 3$ & $4 \frac{3}{4}$ & 20 & $O(5)+O(5)$ \\
\hline
\end{tabular}

\section{СПИСОК ЛИТЕРАТУРЫ}

[1] С. С. Рышков, Е. П. Барановский // Труды МИАН. 1976. Т. 137. [2] С. С. Рышков // Докл. АН ССCP. 1967. Т. 175. № 2. C. 303-305. [3] R. P. Bambah, N. J. A. Sloane // Acta Arith. 1982. V. 42. P. 107-109. [4] W. D. Smith. Studies in Computational Geometry Motivated by Mesh Generation // Ph.D. Dissertation. Dept. of Applied Mathematics, Princeton Univ., 1988. [5] E. P. Baranovskii // European J. Combin. 1994. V. 15. № 4. P. 317-323. [6] H. S. M. Coxeter // Canad. J. Math. 1951. V. 3. P. 391-441. [7] C. С. Рышков // Записки научн. семин. ЛОМИ. 1973. Т. 33. С. 65-71.

Принято редколлегией 23.01.2002 